\title{
Identification of patients at risk of non-adherence to oral antirheumatic drugs in rheumatoid arthritis using the Compliance Questionnaire in Rheumatology: an ARCO sub-study
}

\author{
Carlos Marras ${ }^{1}$, Indalecio Monteagudo ${ }^{2}$, Georgina Salvador ${ }^{3}$, Francisco J. de Toro ${ }^{4}$, \\ Alejandro Escudero ${ }^{5}$, Juan J. Alegre-Sancho ${ }^{6}$, Enrique Raya ${ }^{7}$, Ana Ortiz ${ }^{8}$, Loreto \\ Carmona $^{9}$, Yvonne Mestre ${ }^{10}$, Luis Cea-Calvo ${ }^{10}$, Jaime Calvo-Alén ${ }^{11}$
}

1. Hospital Universitario Virgen de Arrixaca, Calle Polideportivo, 5, 30120 Murcia, Spain

2 Hospital Universitario Gregorio Marañón, Madrid, Spain

3 Hospital Universitario Mutua de Terrassa, Barcelona, Spain

4 Hospital Universitario A Coruña, A Coruña, Spain

5 Hospital Universitario Reina Sofía, Córdoba, Spain

6 Hospital Universitario Dr Peset, Valencia, Spain

7 Hospital Universitario San Cecilio, Granada, Spain

8 Hospital Universitario La Princesa, IIS La Princesa, Madrid, Spain

9 Instituto de Salud Musculoesquelética, Madrid, Spain

10 Medical Affairs, Merck Sharp \& Dohme, Madrid, Spain

11 Hospital Txagorritxu, Araba, Vitoria, Spain

\begin{abstract}
The ARCO study (Study on Adherence of Rheumatoid Arthritis patients to SubCutaneous and Oral Drugs), a multicenter, non-interventional retrospective study, was primarily designed to assess the percentage of patients [aged $\geq 18$ years with an established rheumatoid arthritis (RA) diagnosis] with non-adherence to prescribed subcutaneous biologicals. This paper reports data for the secondary objective from a subset of patients, namely to evaluate nonadherence to prescribed oral antirheumatic drugs in RA patients in Spain using the validated Compliance Questionnaire Rheumatology (CQR). Patients also completed the Morisky-Green Medication Adherence Questionnaire, Beliefs about Medicines Questionnaire, and a questionnaire (developed and validated in Spain) on patient satisfaction with RA treatment and preferences. A total of 271 patients (76.7\% females; mean age 55.6 years) were being treated with oral drugs for RA, of which 234 completed the CQR questionnaire. Non-adherence was reported in 49/234 (20.9\%) patients. The proportion of non-adherence in younger patients (aged $\leq 48$ years; $37.5 \%$ ) was double that recorded in patients aged $>48$ years $(p=0.006)$. Patients with a perception of lower efficacy also had a higher risk of non-adherence $(p=0.012)$. Multivariable analysis showed that younger age and male gender were independently associated with risk of non-adherence. There was only slight agreement between the CQR and Morisky-Green assessment tools (kappa coefficient $=0.186$ ), possibly reflecting the fact that both questionnaires measure slightly different aspects of medication adherence. In conclusion, one out of five RA patients was identified as at risk for non-adherence with the $\mathrm{CQR}$, and this was more frequent in younger patients and in males.
\end{abstract}

Keywords

Adherence; Non-adherence; Oral medication; Questionnaire; Rheumatoid arthritis 


\section{Introduction}

The World Health Organization defines adherence to long-term therapy as the extent to which a person's behavior - taking medication, following a diet, and/or executing lifestyle changes, corresponds with agreed recommendations from a health care provider' [1]. Unfortunately, many patients stop taking their medication, often in the first months following initiation and often without informing their provider $[1,2,3]$. In addition, many patients who continue their medication do not consistently take it as prescribed. Consequently, adherence to long-term therapy for chronic illnesses in developed countries is estimated to average approximately $50 \%[1,2,3,4]$.

Non-adherence is associated with an increased risk for poor health, adverse clinical events, mortality, and greater health care costs in patients with chronic diseases. Indeed, it has been speculated that a reduction in non-adherence may have a greater impact on health than the improvements that can be achieved by the conventional medical treatments [5]. Consequently, the individual patient benefit that could be gained from medication adherence is not achieved, undermining some of the investment in health research and health care. On a global scale, these direct consequences restrict the ability of health care systems to reach population health goals [6].

A range of factors can contribute to poor medication adherence, including patient-related (e.g., suboptimal health literacy and lack of involvement in the treatment decision-making process), physicianrelated (e.g., prescription of complex drug regimens, communication barriers, ineffective communication of information about adverse effects, and provision of care by multiple physicians), and health care system-related (e.g., office visit time limitations, limited access to care, and lack of health information technology) $[7,8]$.

Adherence to medication in patients with rheumatoid arthritis is generally low, varying from 30 to $80 \%$ [8], reflecting that seen in other patient populations. Unfortunately, despite recognizing that medication non-adherence is a common problem, the use of different definitions, designs, populations, treatments, and measurement methods currently precludes solid conclusions from being made regarding the magnitude of non-adherence to medication in rheumatic diseases [8].

We have previously reported non-adherence to prescribed subcutaneous biologicals in patients with rheumatoid arthritis in Spain [9]. In the current descriptive work, using the Compliance Questionnaire in Rheumatology (CQR), we assess the extent of non-adherence to oral antirheumatic drugs in a group of rheumatoid arthritis patients treated with both oral antirheumatic drugs and subcutaneous biological drugs.

\section{Patients and methods}

The ARCO study (Study on Adherence of Rheumatoid Arthritis patients to SubCutaneous and Oral Drugs) was a multicenter, non-interventional retrospective study involving 42 rheumatology clinics of representative hospitals throughout Spain. The study was approved by the corresponding clinical research ethics committees. Patients were recruited between May 2014 and September 2015. The primary objective of the ARCO study was to evaluate adherence to subcutaneous biological drugs, and this has been described elsewhere [9]. As a secondary objective, the study comprised an assessment of the risk of non-adherence to oral antirheumatic drugs, using the CQR, and potential associated factors. As the CQR was validated to assess adherence to antirheumatic medication in general, not at the level of an individual drug, it is important to acknowledge that adherence to specific, individual oral medication was not assessed in this study. 


\section{Selection of participating subjects}

Patients, aged $\geq 18$ years, from rheumatology hospital clinics, with an established diagnosis of rheumatoid arthritis according to EULAR-ACR 2010 criteria and who had started a new subcutaneous biological drug 12-18 months before the study, were screened consecutively and invited to participate in the study. All patients provided signed informed consent prior to study participation. Patients were excluded if they rejected participation, had mental disorders or linguistic difficulties preventing adequate understanding and completion of questionnaires, or serious or unfavorable status precluding study participation, in the judgment of the investigator, or were participating in other studies and/or clinical trials at enrolment or during the retrospective study period.

\section{Procedures}

After inclusion of the subject in the study, data collection was carried out based on a direct interview, physical examination, and review of the medical history. Demographic data, educational level, working status, smoking status, and risk of alcoholism (AUDIT questionnaire) were recorded. Assessment of rheumatoid arthritis activity was made through the DAS-28 calculated using the erythrocyte sedimentation rate (preferably) or C-reactive protein concentration. With regard to medication used for the treatment of RA, data relating to the number of different oral medications and the total number of pills per day were collected. Specific names of active principles were not collected. In line with the study inclusion criteria, all participants were being treated with subcutaneous biological drugs. Comorbidities were classified according to ICD-9 code (International Classification of Diseases).

\section{Evaluation of the risk of non-adherence to oral antirheumatic drugs}

To evaluate non-adherence to oral antirheumatic drugs, use was made of the CQR, a questionnaire that has been developed in patients with rheumatic diseases [10] and validated in rheumatoid arthritis patients compared with an electronic medication event monitoring system [11]. The CQR consists of 19 questions in which patients are asked how much they agree with different statements. Responses are based on Likert scales with a score from 1 to 4 (1: do not agree at all; 2: do not agree; 3: agree; 4: agree very much). The final score allows the identification of non-adherent patients (defined as 'good taking compliance' $\leq 80 \%$ ) with a sensitivity of $62 \%$ and a specificity of $95 \%$ [11]. The CQR has been translated and validated in Spanish [12]. Patients also responded to the Morisky-Green Medication Adherence Questionnaire (4-item version) [13] and completed the Spanish version of the Beliefs about Medicines Questionnaire (BMQ) [14], and a questionnaire (developed and validated in Spain) on patient satisfaction with rheumatoid arthritis treatment and preferences [15].

\section{Statistical analysis}

To describe the sample, measures of central tendency and dispersion [mean, median, standard deviation (SD), interquartile range (IQR)] for continuous variables, and distribution of percentages for categorical variables were used. The Student's $t$ test or ANOVA was used to compare continuous variables (expressed as mean $\pm \mathrm{SD}$ ), while categorical variables were compared using the Chi-squared test. The Chi-squared test, linear trend Chi-squared test, or Fisher exact test was used for comparing proportions.

Adherence was evaluated with the CQR, with an $80 \%$ cut-off point to define potential lack of adherence. The percentage of non-adherence is provided with the $95 \%$ confidence interval $(95 \% \mathrm{CI})$. A logistic regression analysis was used to determine the variables associated with lack of adherence. Odds ratio (OR) and 95\% CI are provided. The performance of the 4-item Morisky-Green adherence scale was compared against the CQR. For this purpose, the results of the 4-item Morisky-Green adherence scale were dichotomized to "high" or "moderate-low" adherence, and compared to the CQR results (adherent 
versus non-adherent patients). The percentage of agreement and the Cohen's kappa coefficient were calculated to correct for the agreement expected by chance, with the following interpretation: poor $(<0)$, slight (0-0.20), fair (0.21-0.40), moderate (0.41-0.60), substantial (0.61-0.80), and almost perfect $(0.81-$ 0.99). No imputation was done for missing data. The $R$ version 3.1.3 Statistical Package was used for statistical analysis.

\section{Results}

Descriptive data

A total of 271 patients [208 female $(76.7 \%)$ and 63 male (23.3\%), mean age 55.6 years (SD 12.0)] were being treated with oral drugs for rheumatoid arthritis at the study visit and completed at least part of the CQR. Demographic characteristics and comorbidities are summarized in Table 1 . The median duration of rheumatoid arthritis since diagnosis was 8.7 years (IQR 25-75: 3.7-16.6). All patients were being treated with subcutaneous biological drugs and, including subcutaneous non-biological drugs, with a median of two non-biological drugs for rheumatoid arthritis (IQR 25-75: 2-3). The median number of pills for rheumatoid arthritis per day was two (IQR 25-75: 1-3). Overall, $44.2 \%$ of patients were in remission (DAS-28<2.6), $18.5 \%$ had low disease activity $(2.6$ to $<3.2), 32.8 \%$ moderate activity (3.2 to $<5.1)$, and $4.5 \%$ high disease activity $(\geq 5.1)$. Almost three-quarters of all patients $(73.8 \%)$ reported at least one comorbid condition, with a median of two concomitant diseases other than rheumatoid arthritis (IQR 25-75: 0-3). 
Table 1. Demographic characteristics of the sample

\begin{tabular}{|c|c|c|}
\hline Characteristic & Mean & SD \\
\hline \multirow[t]{2}{*}{ Age } & 55.6 & 12.0 \\
\hline & Number & Proportion $(\%)$ \\
\hline \multicolumn{3}{|l|}{ Gender } \\
\hline Male & 63 & 23.3 \\
\hline Female & 208 & 76.7 \\
\hline \multicolumn{3}{|l|}{ Race } \\
\hline Caucasian & 254 & 93.7 \\
\hline \multicolumn{3}{|l|}{ Smoking ${ }^{\mathrm{a}}$} \\
\hline Smoker & 70 & 26.5 \\
\hline Ex-smoker & 131 & 49.6 \\
\hline Never-smoker & 63 & 23.9 \\
\hline \multicolumn{3}{|l|}{ Alcoholism risk (AUDIT) } \\
\hline Yes & 19 & 7.0 \\
\hline \multicolumn{3}{|l|}{ Educational level } \\
\hline Primary school & 130 & 48.0 \\
\hline Secondary school (graduated) & 55 & 20.3 \\
\hline Professional studies & 51 & 18.8 \\
\hline University degree & 35 & 12.9 \\
\hline \multicolumn{3}{|l|}{ Working status } \\
\hline Currently working (out of home) & 105 & 38.7 \\
\hline Home care & 38 & 14.0 \\
\hline Unemployed & 28 & 10.3 \\
\hline Retired & 78 & 28.8 \\
\hline Disabled for work & 20 & 7.4 \\
\hline Student & 2 & 0.7 \\
\hline
\end{tabular}

AUDIT alcohol use disorders identification test, $S D$ standard deviation

${ }^{\text {a }}$ Data not reported for seven patients

Non-adherence to oral antirheumatic drugs and relationship to demographic and clinical characteristics

From the 271 patients, 37 were excluded from the non-adherence analysis, because the CQR questionnaire was not complete. There were no differences between the characteristics of the 234 patients with a complete CQR and the excluded patients. Forty-nine patients had CQR results suggesting nonadherence (prevalence 20.9\%; 95\% CI 16.2-26.6). Items from the CQR with significant differences in the responses between adherent and non-adherent patients were the following: "I definitely do not dare to miss my antirheumatic medications" $(p<0.001)$, "My medicines are always stored in the same place, and that is why I do not forget them" ( $p<0.001)$, "I take my medicines, because I have complete confidence in my rheumatologist" $(p=0.019)$, "When I am on vacation, it sometimes happens that I do not take my medicines" $(p=0.005)$, "If you cannot stand the medicines you might say: throw it away, no matter what" $(p=0.047)$, "If I do not take my antirheumatic medicines, my body warns me" $(p=0.004)$, "My health goes above everything else and if I have to take medicines to keep well, I will" ( $p=0.005)$, "I use a dose organizer for my medications" $(p=0.011)$, "What the doctor tells me, I hang on to" $(p=0.003)$, and "It happens every now and then, I go out for the weekend and then I do not take my medicines" $(p<0.001)$. Specific responses on the CQR are shown in the Supplementary Material. 
The proportion of non-adherence in younger patients (aged $\leq 48$ years; 37.5\%) was double that recorded in patients aged $>48$ years (Table 2). Non-Caucasian patients and those actively working also showed higher percentages of non-adherence, although the differences did not reach statistical significance, and the percentage was also numerically higher in males (Table 2). There were no differences in the percentage of non-adherence by other sociodemographic factors, such as level of education, smoking habit, or alcohol intake.

Table 2. Percentage of patients who were non-adherent to oral antirheumatic drugs, by demographic characteristics

\begin{tabular}{|c|c|c|}
\hline & Non-adherence $(\%)$ & $p$ value \\
\hline \multicolumn{3}{|l|}{ Age } \\
\hline Q1 $(\leq 48$ years old $)(n=56)$ & 37.5 & 0.006 \\
\hline Q2 $(>48$ to $\leq 56$ years old $)(n=64)$ & 15.6 & \\
\hline Q3 $(>56$ to $\leq 63$ years old $)(n=59)$ & 13.6 & \\
\hline Q4 $(>63$ years old $)(n=55)$ & 18.2 & \\
\hline \multicolumn{3}{|l|}{ Gender } \\
\hline Male $(n=55)$ & 27.3 & 0.258 \\
\hline Female $(n=179)$ & 19.0 & \\
\hline \multicolumn{3}{|l|}{ Race } \\
\hline Caucasian $(n=219)$ & 19.6 & 0.093 \\
\hline Other race $(n=15)$ & 40.0 & \\
\hline \multicolumn{3}{|l|}{ Smoking habit } \\
\hline Smoker $(n=60)$ & 16.7 & 0.232 \\
\hline Ex-smoker $(n=110)$ & 18.2 & \\
\hline Never-smoker $(n=57)$ & 28.1 & \\
\hline \multicolumn{3}{|l|}{ Alcoholism risk (AUDIT) } \\
\hline Yes $(n=19)$ & 26.3 & 0.559 \\
\hline No $(n=215)$ & 20.5 & \\
\hline \multicolumn{3}{|l|}{ Educational level } \\
\hline Primary school $(n=112)$ & 16.1 & 0.345 \\
\hline Secondary school (graduated) $(n=47)$ & 27.7 & \\
\hline Professional studies $(n=41)$ & 24.4 & \\
\hline University degree $(n=34)$ & 23.5 & \\
\hline \multicolumn{3}{|l|}{ Working status } \\
\hline \multirow[t]{2}{*}{ Currently working $(n=90)$} & 27.8 & 0.062 \\
\hline & 16.7 & \\
\hline
\end{tabular}

AUDIT alcohol use disorders identification test

With regard to disease characteristics, the percentages of non-adherence were similar in those with rheumatoid arthritis duration above/below the median $(21.4$ and $20.0 \%$, respectively, $p=0.918)$, in those treated with/without concomitant non-biological subcutaneous drugs $(25.8$ and $19.2 \%, p=0.360)$, or in those with different amounts of rheumatoid arthritis medication per day (Fig. 1). Although not statistically significant, patients with no comorbidities showed a higher percentage of non-adherence than those with at least one comorbidity $(30.2$ vs. $17.5 \%, p=0.055)$, and the percentage of non-adherence was lower in those taking a higher number of pills per day overall $(p=0.084$; Fig. 1$)$. There were no differences in the percentage of non-adherent patients with regard to the results of the $\mathrm{BMQ}$, nor with regard to the overall 
perceived treatment tolerability, but non-adherence was higher in those who declared that the effect of the treatment on rheumatoid arthritis symptoms had been less than expected ( $p=0.012 ;$ Fig. 2$)$.

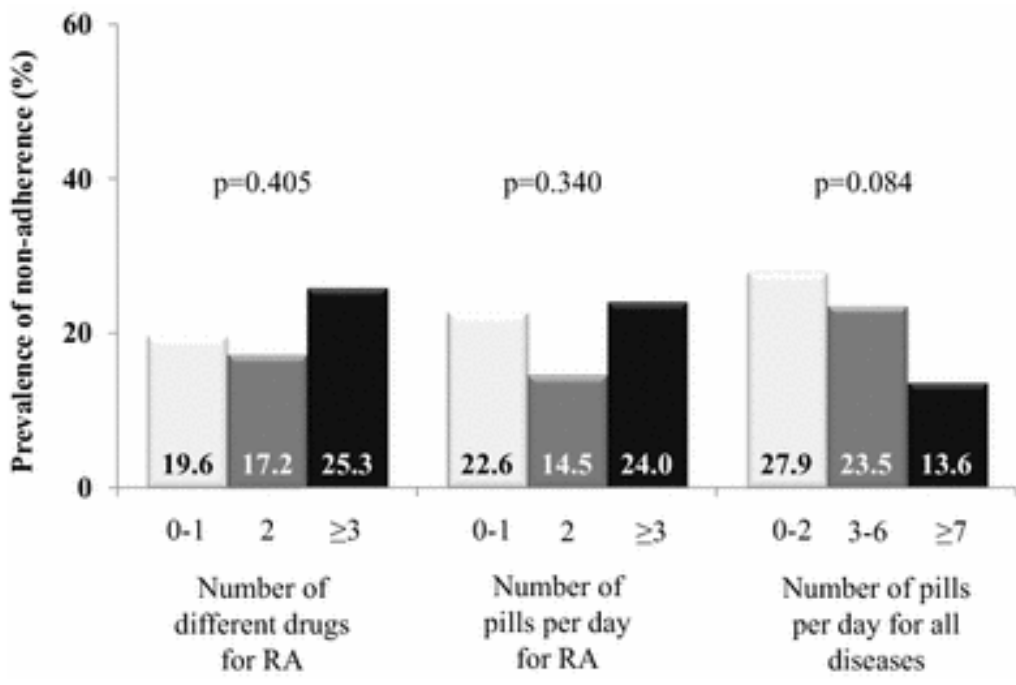

Fig. 1. Proportion of non-adherent rheumatoid arthritis (RA) patients, stratified by the number of different drugs for RA, the number of pills per day for RA, and the number of pills per day for all diseases

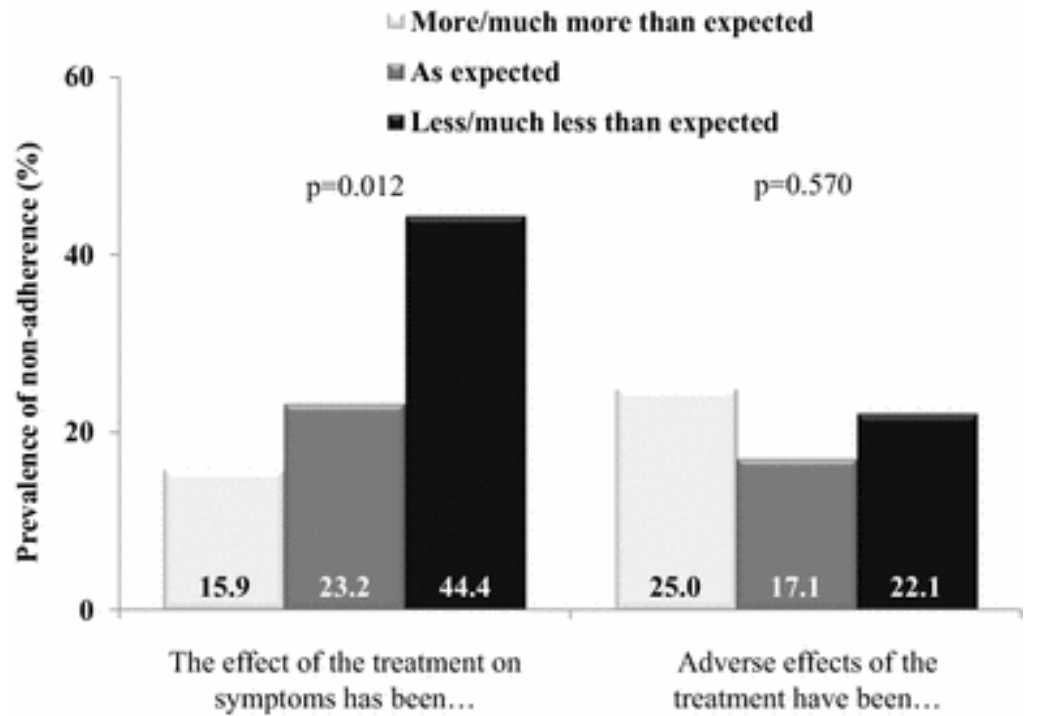

Fig. 2. Impact of rheumatoid arthritis medication efficacy and tolerability on nonadherence 
Disease activity was similar among adherent and non-adherent patients [mean DAS 28: $2.8(1.2)$ and 2.9 (1.3), respectively, $p=0.659$ ]. Likewise, the classification into disease remission (48.3 and 44.9\%), low (18.9 and $14.3 \%)$, moderate (28.9 and 38.8\%), or high (3.9 and 2.0\%) disease activity was similar in adherent versus non-adherent patients.

Variables associated with non-adherence were assessed using a multivariable model that included age, gender, race, rheumatoid arthritis duration, working status, concomitant diseases, and pills per day (Table 3). The model identified younger age, male gender, non-Caucasian race, and rheumatoid arthritis duration, as variables with the highest OR for non-adherence (Table 3a), and the final model identified younger age as independently associated with non-adherence (Table 3b). Male gender points to an association too, but given the low number of males included in the study, a significant $p$ value was not reached in the multivariate regression analysis.

Table 3. Multivariable analysis

\begin{tabular}{llll}
\hline & Odds ratio $95 \%$ CI & $p$ value \\
\hline & & & \\
a. Initial logistic regression model & & & \\
Age (per year of increase) & 0.96 & $0.93-0.99$ & 0.025 \\
Male gender (vs. female) & 2.00 & $0.93-4.35$ & 0.074 \\
Race (non-Caucasian vs. Caucasian) & 2.12 & $0.61-7.06$ & 0.221 \\
Working status (currently working vs. other status) & 1.04 & $0.48-2.27$ & 0.911 \\
RA duration above median (>7.8 years) & 1.73 & $0.85-3.60$ & 0.136 \\
Concomitant diseases (yes vs. no) & 0.78 & $0.36-1.74$ & 0.543 \\
Number of pills per day(>6 vs. $\leq 6)$ & 0.64 & $0.27-1.43$ & 0.282 \\
b. Final model & & & \\
Age (per year of increase) & 0.95 & $0.93-0.98<0.001$ \\
Male gender (vs. female) & 1.96 & $0.93-4.17$ & 0.070 \\
& & & \\
\hline
\end{tabular}

Variables associated with non-adherence

$R A$ rheumatoid arthritis

\section{Non-adherence and results of the Morisky-Green test}

Based on the 4-item Morisky-Green test, 164 patients (70.1\%) had high adherence and 70 (29.9\%) were non-adherent [69 (29.5\%) had moderate adherence and $1(0.4 \%)$ had low adherence]. The percentage of non-adherence with the CQR was significantly higher in those classified as non-adherent with the Morisky-Green ( $p=0.009$; Fig. 3). The percentage of agreement between the Morisky-Green test and the CQR was $68.8 \%$, and the kappa coefficient showed only a slight agreement (kappa $=0.186$, $95 \%$ CI $0.031-0.341, p=0.012)$. 


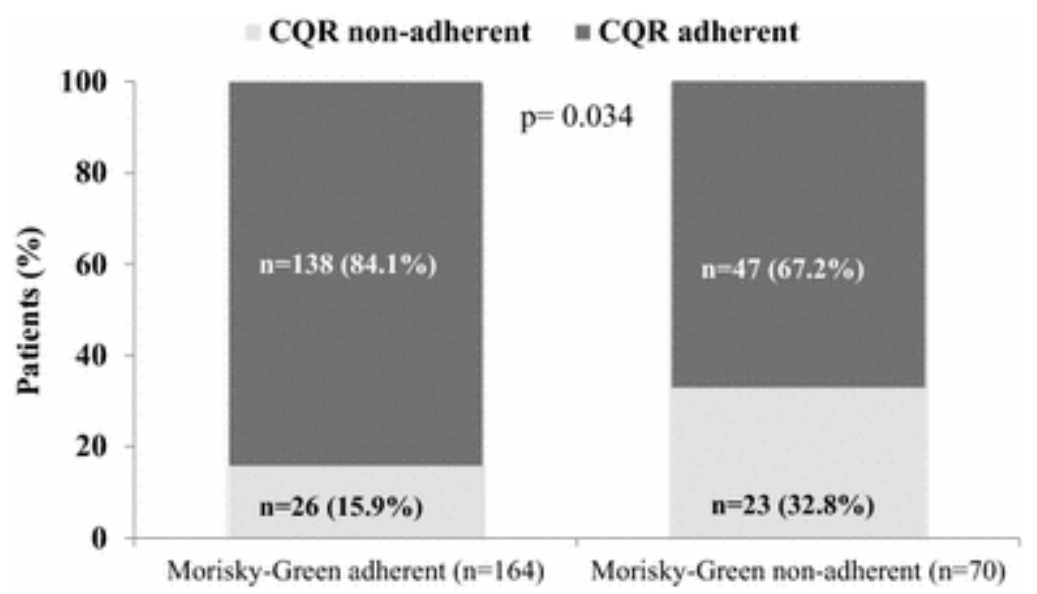

Fig. 3. Adherence/non-adherence assessed using the Compliance Questionnaire in Rheumatology (CQR) and the Morisky-Green Medication Adherence Questionnaire (4-item version)

\section{Discussion}

The current analysis from a large subset of patients included in the ARCO study in Spain shows that non-adherence to oral antirheumatic drugs occurred in $20.9 \%$ of patients, according to the CQR. Multivariable analysis revealed that younger age and male gender were independently associated with non-adherence in this population.

There is currently no optimal method to assess adherence to oral drugs. Indeed, numerous different methods are available to assess medication adherence, including subjective (e.g., self-reporting, physicians' assessment), direct (e.g., biomarkers), or indirect (e.g., pharmacy refill, tablet counts, electronic monitors, questionnaires) measurements, each with potential advantages or disadvantages [4, 8]. Advantages of self-report medication adherence questionnaires include their ease of use and the fact that they are noninvasive and can be validated against other assessment tools. Key disadvantages of questionnaires are that they can be susceptible to error due to increases in time between visits, and the fact that the patient is aware of the measurement and, therefore, may answer based on expectation rather than reality $[4,8]$.

Several previous studies have used the CQR to assess non-adherence, and possible factors associated with non-adherence, to oral rheumatoid arthritis medication [16, 17, 18]. In the largest of these studies, non-adherence to disease-modifying antirheumatic drugs (DMARDs) was assessed in 228 randomly selected patients with rheumatoid arthritis [16]. In this population, $32-40 \%$ of the patients did not adhere to their DMARD prescription. As none of the evaluated potential risk factors (including demographic and clinical characteristics, satisfaction about information, medication concerns, coping styles, disease duration, the number of perceived side effects, and beliefs about the necessity for the medicine) were strongly related to adherence, the study authors concluded that no general risk factor appears to be powerful enough as a possible screening tool or target for adherence-improving interventions [8]. A strong perception of a personal need for the treatment was associated with improved adherence in a study which specifically assessed adherence to methotrexate in 126 patients with rheumatoid arthritis. Medication adherence did not appear to be influenced by the patients' functional impairment or the methotrexate dose level [17]. In contrast, a recent observational study which investigated adherence to methotrexate (orally or via injection) in 129 patients with rheumatoid arthritis, reported that a better mental health status and experiencing comorbidities had a positive effect on adherence, while living alone had a negative effect [18]. Taken together, the findings from these studies and the current study (all utilizing the CQR), in addition to data from recent literature reviews [19, 20], indicate that, whilst there is 
overlap in some of the possible determinants of non-adherence across various studies, no clear pattern emerges with regard to risk factors associated with non-adherence to oral medication in patients with rheumatoid arthritis. Indeed, the general implication is that because potential barriers to medication adherence are complex and varied, they should be evaluated on an individual basis, and solutions to improve adherence must be multifactorial [7].

Data from a systematic review of the impact of medication regimen factors on adherence to chronic treatment showed that in most of the therapeutic areas evaluated (with the exception of asthma/pulmonary disease), there is some evidence that greater dosing frequency is associated with poorer adherence. Indeed, across a range of chronic diseases, including diabetes, hypertension, and HIV/AIDS, the authors found strong, consistent evidence that increases in dose frequency and regimen complexity (multiple medications, multiple doses, specific dietary, or time requirements) are related to poorer adherence [21]. Interestingly, our study showed no significant difference in non-adherence rates to oral antirheumatic drugs in patients treated with/without concomitant non-biological subcutaneous drugs, or in those with different amounts of rheumatoid arthritis medication per day, or in those taking a higher number of pills per day for rheumatoid arthritis or all diseases. The reason for this finding is not clear, but as the CQR questions patients about general attitudes or customs towards taking antirheumatic medicines, those with multiple morbidities or those receiving polymedication might be more prone to respond positively to such questions and have less risk of non-adherence based on the established $80 \%$ cut-off value. In addition, the population selected for the current study (i.e., patients also treated with subcutaneous biological drugs) could have resulted in a selection bias towards a population which was sicker, and more aware of the seriousness of their disease and of the importance of fulfilling treatment schedules. These factors may also account for the relatively low percentage of patients at risk of non-adherence identified in the current study, and for the lack of differences in disease activity between adherent and non-adherent patients. Patients were required to be on the same biological drug for 12 months to qualify for the study, and this might have conditioned a selection bias towards a population with better disease control despite nonadherence.

Our study showed only slight agreement between the CQR and the Morisky-Green questionnaire. This may reflect the fact that both questionnaires measure slightly different aspects of medication adherence: the Morisky-Green scale asks patients directly whether they forgot or missed doses, whereas the CQR requests information on attitudes or behaviors that can indicate a risk for non-adherence. It is also worth noting that the Morisky-Green test is a general medication adherence assessment tool [13], whereas the CQR has been developed and validated specifically for use in patients with inflammatory rheumatic diseases [11]. Further studies should explore whether both questionnaires complement each other in the identification of patients at risk of non-adherence.

The current study has some limitations. The CQR makes an assessment of adherence to oral drugs globally, not at the level of an individual drug. As adherence to one particular oral antirheumatic may be different from another, it is important to acknowledge that adherence to individual oral drugs (including glucocorticoids) was not assessed in our study. Indeed, it has been noted recently that in addition to understanding the validity of the assessment method used, and the scales and cut-off points, an appraisal of adherence studies needs to be done according to the medications evaluated [20]. Selection bias may also represent a limitation of our study. Patients were required to have been prescribed a new subcutaneous biological drug 12-18 months before the study visit, so it is possible that patients with a better response to the overall medication were selected into the ARCO study. Patients who failed to respond to the biological medication, or who lost response, were not likely to be included in the study, because they were likely to have been switched to other drugs prior to the study visit. It must also be acknowledged that $14 \%$ of patients were excluded from the analysis of oral medication adherence because of incomplete CQRs; the impact, if any, of these excluded patients on the overall adherence data remains unknown. 
In conclusion, using the CQR, the current analysis from a subset of patients included in the ARCO study in Spain shows that non-adherence to oral antirheumatic drugs occurred in $20.9 \%$ of patients. Younger age and male gender were independently associated with non-adherence in this population. The CQR is a useful tool that can help to identify patients at risk for non-adherence, through the information collected with regard to patients' attitudes or behaviors towards taking their antirheumatic medication.

Acknowledgements. Study implementation and statistical analysis were conducted by Experior S.L, Spain. Medical writing assistance was provided by David P. Figgitt Ph.D., Content Ed Net, with funding from Merck Sharp \& Dohme, Spain. The authors would like to thank the 42 study investigators for their contribution to patient recruitment and data acquisition, and to the patients involved for their collaboration in completing the study questionnaires.

\section{Compliance with ethical standards}

Conflict of interest. The ARCO study was financed by Merck Sharp \& Dohme, Spain, a subsidiary of Merck \& Co, Kenilworth, New Jersey, USA. Yvonne Mestre and Luis Cea-Calvo are full-time employees of Merck Sharp \& Dohme (MSD), Spain. The co-authors of the manuscript were also study investigators, and they or their institution received financial compensation for the enrolment of patients in the study. Georgina Salvador has received payments for lectures from BMS, Roche, AbbVie, Amgen, and Pfizer, and for the development of educational presentations from BMS, Roche, Pfizer, AbbVie, and MSD. Juan J. Alegre has received payments as a board member from AbbVie; for consultancy from MSD; for his expert testimony from Roche; for lectures from AbbVie, MSD, Roche, BMS, and Pfizer; for the development of educational presentations from BMS; and his institution has received grants from AbbVie. Ana Ortiz has received payments for grants from the "Instituto de Salud Carlos III". L. Carmona's institution (InMusc.) has received consultancy honoraria from MSD. The rest of the authors declare no other conflicts of interest.

\section{References}

1. World Health Organization (WHO). Adherence to long-term therapies: evidence for action. Geneva, Switzerland. 2003.

http://www.who.int/chp/knowledge/publications/adherence_full_report.pdf. Accessed 25 July 2016

2. Gialamas A, Yelland LN, Ryan P, Willson K, Laurence CO, Bubner TK, Tideman P, Beilby JJ (2009) Does point-of-care testing lead to the same or better adherence to medication? A randomised controlled trial: the PoCT in General Practice Trial. Med J Aust 191(9):487-491

3. Naderi SH, Bestwick JP, Wald DS (2012) Adherence to drugs that prevent cardiovascular disease: meta-analysis on 376,162 patients. Am J Med 125(9):882.e1-887.e1

4. Osterberg L, Blaschke T (2005) Adherence to medication. N Engl J Med 353(5):487-497

5. Haynes RB, Ackloo E, Sahota N, McDonald HP, Yao X (2008) Interventions for enhancing medication adherence. Cochrane Database Syst Rev 2:CD000011

6. Nieuwlaat R, Wilczynski N, Navarro T, Hobson N, Jeffery R, Keepanasseril A, Agoritsas T, Mistry N, Iorio A, Jack S, Sivaramalingam B, Iserman E, Mustafa RA, Jedraszewski D, Cotoi C, Haynes RB (2014) Interventions for enhancing medication adherence. Cochrane Database Syst Rev 11:CD000011

7. Brown MT, Bussell JK (2011) Medication adherence: WHO cares? Mayo Clin Proc 86(4):304314

8. van den Bemt BJ, Zwikker HE, van den Ende CH (2012) Medication adherence in patients with rheumatoid arthritis: a critical appraisal of the existing literature. Expert Rev Clin Immunol 8(4):337-351

9. Calvo-Alén J, Monteagudo I, Salvador G et al (2016) Non-adherence to subcutaneous biological medication in patients with rheumatoid arthritis: a multicentre, non-interventional study. Clin Exp Rheumatol 34:1-8 
10. de Klerk E, van der Heijde D, van der Tempel H, van der Linden S (1999) Development of a questionnaire to investigate patient compliance with antirheumatic drug therapy. J Rheumatol 26(12):2635-2641

11. de Klerk E, van der Heijde D, Landewé R, van der Tempel H, van der Linden S (2003) The compliance-questionnaire-rheumatology compared with electronic medication event monitoring: a validation study. J Rheumatol 30(11):2469-2475

12. Arturi P, Schneeberger EE, Sommerfleck F, Buschiazzo E, Ledesma C, Maldonado Cocco JA, Citera G (2013) Adherence to treatment in patients with ankylosing spondylitis. Clin Rheumatol 32(7):1007-1015

13. Morisky DE, Green LW, Levine DM (1986) Concurrent and predictive validity of a selfreported measure of medication adherence. Med Care 24:67-74

14. Beléndez-Vázquez M, Hernández-Mijares A, Horne R, Weinman J (2007) Evaluación de las creencias sobre el tratamiento: validez y fiabilidad de la versión española del "Beliefs about Medicines Questionnaire" [Assessment of beliefs about treatment: validity and reliability of the Spanish version of the "Beliefs about Medicines Questionnaire"]. Int J Clin Health Psychol 7:767-779 (article in Spanish)

15. Carbonell J, Badia X, Grupo EXPRESAR (2006) Desarrollo y validación de un cuestionario de satisfacción con el tratamiento en pacientes con artritis reumatoide. [Development and validation of a satisfaction questionnaire in patients with rheumatoid arthritis]. Reumatol Clin 2(3):137-145 (article in Spanish)

16. van den Bemt BJ, van den Hoogen FH, Benraad B, Hekster YA, van Riel PL, van Lankveld W (2009) Adherence rates and associations with nonadherence in patients with rheumatoid arthritis using disease modifying antirheumatic drugs. J Rheumatol 36(10):2164-2170

17. de Thurah A, Nørgaard M, Harder I, Stengaard-Pedersen K (2010) Compliance with methotrexate treatment in patients with rheumatoid arthritis: influence of patients' beliefs about the medicine. A prospective cohort study. Rheumatol Int 30(11):1441-1448

18. De Cuyper E, De Gucht V, Maes S, Van Camp Y, De Clerck LS (2016) Determinants of methotrexate adherence in rheumatoid arthritis patients. Clin Rheumatol 35(5):1335-1339 19.

19. Pasma A, van't Spijker A, Hazes JM, Busschbach JJ, Luime JJ (2013) Factors associated with adherence to pharmaceutical treatment for rheumatoid arthritis patients: a systematic review. Semin Arthritis Rheum 43(1):18-28

20. Scheiman-Elazary A, Duan L, Shourt C, Agrawal H, Ellashof D, Cameron-Hay M, Furst DE (2016) The rate of adherence to antiarthritis medications and associated factors among patients with rheumatoid arthritis: a systematic literature review and metaanalysis. J Rheumatol 43(3):512-523

21. Ingersoll KS, Cohen J (2008) The impact of medication regimen factors on adherence to chronic treatment: a review of literature. J Behav Med 31(3):213-224 